\title{
The evolutionary characteristics of missing proteins: \\ Insights into the evolution of chromosomes related to missing-protein-encoding genes \\ (Supporting information)
}

\section{Contents}

\section{Supplementary description of part of the results}

- Supplementary description of the missing-protein-encoding gene distribution in telomeres and centromeres

- Supplementary description of the functional characteristics of the proteins with extremely special physical-chemical properties

- Supplementary description of the identification status of OR genes' products in neXtProt database

- Supplementary description of the OR genes that were identified in the transcriptome, translatome and proteome datasets of previous studies

\section{Supplementary tables}

Table S1. Clusters contain more than 5 genes and at least 1 missing-protein-coding gene.

Table S2. Detailed information of all the genes in paralogous gene groups (PGGs) (in the file “Supporting Information - Table S2, Table S6 and Table S7.xlsx" ).

Table S3. Orders of genes' duplication time.

Table S4. List of PE3/4 genes originated from the latest common ancestor of Opisthokonta.

Table S5. The number of OR genes in neXtProt database.

Table S6. The number of OR genes identified in the transcriptome, translatome and proteome datasets of previous studies (in the file "Supporting Information - Table S2, Table S6 and Table S7.xlsx").

Table S7. Gene Ontology over/under-representation analysis of the proteins with extremely special physical-chemical properties (in the file "Supporting Information - Table S2, Table S6 and Table S7.xlsx").

\section{Supplementary figures}

Figure S1. Schematic diagram for the relative locations of gene and block.

Figure S2. The percentages of the (missing-protein-encoding) genes closed to the telomeres in short arms, center of centromere and telomeres in long arms among all (missing-protein-encoding) genes in each chromosome.

Figure S3. The percentages of the two types of missing-protein-encoding genes closed to the 
telomeres in short arms, center of centromere and telomeres in long arms among all genes in each region.

Figure S4. Ages of singletons.

Figure S5. Evolutionary characteristics of genes encoding PE3 and PE4 proteins.

Figure S6. The cumulative probability curve of the difference values of average order of PE3/4 genes and OTCSGs.

Figure S7. Evolutionary characteristics of the proteins with extremely special physical-chemical properties. 


\section{Supplementary description of the missing-protein-encoding gene distribution in telomeres and centromeres}

(1) In the terminal region of short arm of chromosome11, the percentage of PE2 genes against all the PE2 genes in the chromosome is the largest among all the chromosomes. And the percentage is higher than expectation (5\%) and also higher than the percentages of backgrounds (the percentage of PCGs in this region against all the PCGs in the same chromosome). For PE3/4 genes, in terminal region of short arm of chromosome 4, the percentage of PE3/4 genes against all the PE3/4 genes in the chromosome is the largest among all the chromosomes (Figure S2A).

(2) In the centromere region of chromosome 14, the percentages of both PE2 genes and PE3/4 genes against all the PE2 genes/ PE3/4 genes in the chromosome are the largest among all the chromosomes. And the percentage are higher than expectation (10\%) and also higher than the percentages of backgrounds (Figure S2B).

(3) In the terminal regions of long arms of chromosomes 5, the percentages of PE2 genes against all the PE2 genes in the chromosome is the largest among all the chromosomes. And the percentage is higher than expectation (5\%) and also higher than the percentages of backgrounds. For PE3/4 genes, in terminal region of long arm of chromosome 22, the percentage of PE3/4 genes against all the PE3/4 genes in the chromosome is the largest among all the chromosomes. (Figure S2C).

\section{Supplementary description of the functional characteristics of the proteins with extremely special physical-chemical properties}

The three classes of proteins with extremely special physical-chemical properties tend to have different over-represented GO items for biological processes or cellular components (Table S5). For example, highly hydrophobic proteins (hydrophobicity value $>0.615$ ) mainly distribute in membrane and mainly take part in the processes of "sensory perception of chemical stimulus", " response to stimulus" and "GPCR signaling pathway". The low-molecular-weight proteins (MW<13645.8 Da) tend to localize in macromolecular complexes or in the extracellular regions and take part in the processes of translation, locomotion, and "immune system process". The high-isoelectric-point proteins (isoelectric point $>8.93$ ) have some characteristics same to the 
other two categories, but they also have their own over-represented terms, such as the biological processes of transcription, metabolic process and its regulation, and the localization in nucleus.

\section{Supplementary description of the identification status of OR genes' products in neXtProt database}

To characterize the identification status of OR genes' products, we calculated the number of OR genes in neXtProt database (Release 20140919 and 20150901) (Table S5). As the result, most OR genes encode missing proteins (PE2 or PE3). It is notable that many OR genes previously in PE2 (with transcript evidence) are now considered as PE3 genes (without transcript evidence). According to the newest version of neXtProt (20150901), there are 208 OR genes with transcript evidence (no protein evidence).

\section{Supplementary description of the OR genes that were identified in the transcriptome, translatome and proteome datasets of previous studies}

3 datasets of 8 cell lines with the data of transcriptome, translatome and proteome were employed in our analysis on OR genes (Table S6). Translatome means all the RNAs binding to ribosomes in the cell, representing the genes which are translated in the cell. It's very interesting that among the 399 OR genes of human, 32 transcript of them can be identified, whereas there are only 18 OR genes identified in the corresponding translatome. Other 14 OR genes (43.75\%) probably only had transcripts, but these transcripts weren't translated into proteins. Among the 18 translated genes, only one was detected by shotgun proteomic technology. This phenomenon may be due to the extremely high hydrophobicity of the 17 OR proteins (all of them $>0.615$, the threshold value for extremely high hydrophobicity. Mean value=0.756).

Reference:

Wang, T.; Y. Cui; J. Jin; J. Guo; G. Wang; X. Yin; Q.-Y. He and G. Zhang, Translating mRNAs strongly correlate to proteins in a multivariate manner and their translation ratios are phenotype specific. Nucleic acids research 2013, gkt178.

Chang; C.; L. Li; C. Zhang; S. Wu; K. Guo; J. Zi; Z. Chen; J. Jiang; J. Ma and Q. Yu, Systematic 
analyses of the transcriptome, translatome, and proteome provide a global view and potential strategy for the C-HPP. Journal of proteome research 2013, (1): 38-49.

Zhong; J.; Y. Cui; J. Guo; Z. Chen; L. Yang; Q.-Y. He; G. Zhang and T. Wang, Resolving chromosome-centric human proteome with translating mRNA analysis: a strategic demonstration. Journal of proteome research 2013, (1): 50-59. 
Table S1. Clusters contain more than 5 genes and at least 1 missing-protein-coding gene

\begin{tabular}{|c|c|c|c|c|c|}
\hline Claster & Chromosome & Sites & Description & Gene number & $\begin{array}{r}\text { Missing-protein-encoding } \\
\text { gene number }\end{array}$ \\
\hline 1 & 1 & 152483320 to 152816459 & Late cornified envelope protein & 21 & 14 \\
\hline 2 & 2 & 176942200 to 176997423 & Homeobox protein & 7 & 2 \\
\hline 3 & 2 & 234580499 to 234681945 & UDP-glucuronosyltransferase & 6 & 1 \\
\hline 4 & 4 & 9212383 to 9370796 & Ubiquitin carboxyl-terminal hydrolase & 20 & 12 \\
\hline 5 & 4 & 190992087 to 191013719 & Double homeobox protein & 7 & 7 \\
\hline 6 & 5 & 140165876 to 140391929 & Protocadherin alpha & 13 & 8 \\
\hline 7 & 5 & 140552243 to 140627799 & Protocadherin beta & 10 & 3 \\
\hline 8 & 5 & 140710252 to 140892546 & Protocadherin gamma & 18 & 11 \\
\hline 9 & 7 & 27145803 to 27239725 & Homeobox protein & 9 & 2 \\
\hline 10 & 11 & 1605572 to 1652160 & Keratin-associated protein & 5 & 4 \\
\hline 11 & 11 & 55563032 to 55607214 & Olfactory receptor & 5 & 5 \\
\hline 12 & 13 & 57715052 to 57744352 & Proline-rich protein & 5 & 5 \\
\hline 13 & 17 & 39133968 to 39222131 & Keratin-associated protein & 11 & 4 \\
\hline 14 & & 39344697 to 39471947 & Keratin-associated protein & 11 & 3 \\
\hline 15 & 19 & 49535169 to 49562117 & Choriogonadotropin subunit beta & 5 & 2 \\
\hline 16 & 21 & 31852018 to 31874435 & Keratin-associated protein & 5 & 3 \\
\hline 17 & 21 & 31962424 to 32015455 & Keratin-associated protein & 9 & 5 \\
\hline 18 & 21 & 45993606 to 46117959 & Keratin-associated protein & 13 & 2 \\
\hline 19 & $\mathrm{X}$ & 49160148 to 49242997 & $\mathrm{G}$ antigen & 7 & 6 \\
\hline 20 & $\mathrm{X}$ & 49296814 to 49373139 & $\mathrm{G}$ antigen & 8 & 5 \\
\hline
\end{tabular}


Table S3. Orders of genes' last duplication time. The evolutionary time was represented by the ancestors in the phylogeny tree. Each ancestor was given a matched order based on the divergent time in the phylogeny tree.

\begin{tabular}{|l|r|}
\hline Ancestor & Order \\
\hline Opisthokonta & 20 \\
\hline Bilateria & 19 \\
\hline Chordata & 18 \\
\hline Vertebrata & 17 \\
\hline Euteleostomi & 16 \\
\hline Sarcopterygii & 15 \\
\hline Tetrapoda & 14 \\
\hline Amniota & 13 \\
\hline Mammalia & 12 \\
\hline Theria & 11 \\
\hline Eutheria & 10 \\
\hline Euarchontoglires & 9 \\
\hline Primates & 8 \\
\hline Haplorrhini & 7 \\
\hline Simiiformes & 6 \\
\hline Catarrhini & 5 \\
\hline Hominoidea & 4 \\
\hline Hominidae & 3 \\
\hline Homininae & 13 \\
\hline Homo sapiens & 17 \\
\hline
\end{tabular}


Table S4. List of PE3/4 genes originating from the latest common ancestor of Opisthokonta

\begin{tabular}{|c|c|c|c|}
\hline ENSEMBL ID & Group ID & Nextprot PE & $\begin{array}{c}\text { The last duplication } \\
\text { ancestor }\end{array}$ \\
\hline ENSG00000168255 & 3 & 3 & Homo sapiens \\
\hline ENSG00000223569 & 477 & 3 & Homo sapiens \\
\hline ENSG00000227140 & 477 & 3 & Homo sapiens \\
\hline ENSG00000227551 & 477 & 3 & Homo sapiens \\
\hline ENSG00000231396 & 477 & 3 & Homo sapiens \\
\hline ENSG00000232399 & 477 & 3 & Homo sapiens \\
\hline ENSG00000233136 & 477 & 3 & Homo sapiens \\
\hline ENSG00000248920 & 477 & 3 & Homo sapiens \\
\hline ENSG00000248933 & 477 & 3 & Homo sapiens \\
\hline ENSG00000249104 & 477 & 3 & Homo sapiens \\
\hline ENSG00000249811 & 477 & 3 & Homo sapiens \\
\hline ENSG00000250745 & 477 & 3 & Homo sapiens \\
\hline ENSG00000250844 & 477 & 3 & Homo sapiens \\
\hline ENSG00000188991 & 974 & 3 & Vertebrata \\
\hline ENSG00000183921 & 1101 & 3 & Vertebrata \\
\hline ENSG00000170935 & 1762 & 3 & Eutheria \\
\hline ENSG00000215029 & 2040 & 3 & Homo sapiens \\
\hline ENSG00000196873 & 2376 & 3 & \\
\hline
\end{tabular}


Table S5. The number of OR genes in neXtProt database

\begin{tabular}{|l|c|c|}
\hline \multirow{2}{*}{ Class } & \multicolumn{2}{|c|}{ Number of OR genes in neXtProt database } \\
\cline { 2 - 3 } & Release 20140919 & Release 20150901 \\
\hline PE1 & 8 & 5 \\
\hline PE2 & 357 & 208 \\
\hline PE3 & 38 & 191 \\
\hline PE4 & 0 & 0 \\
\hline PE5 & 19 & 17 \\
\hline
\end{tabular}




\section{Supplementary figure legends}

Figure S1. Schematic diagram for the relative locations of gene and block. Block (blue) means the homologous synteny block defined by the comparison among the genome sequences from different species (See the Method section in the main text for detailed information). There are 4 types of locations for the genes relative to the blocks (In block, Outside of block, Partial in block, Span block).

Figure S2. The percentages of the (missing-protein-encoding) genes closed to the telomeres in short arms (A), center of centromere (B) and telomeres in long arms (C) among all (missing) genes in each chromosome. The cutoff value for the distance of the gene near the center of centromere or the gene near telomeres were $5 \%$ length of each chromosome

Figure S3. The percentages of the two types of missing-protein-encoding genes closed to the telomeres in short arms (A), center of centromere (B) and telomeres in long arms (C) among all genes in each region.

Figure S4. Ages of singletons. All the singletons are divided into 6 groups according to their evolutionary origin time, including the genes originating from the common ancestor of Opisthokonta, Bilateria, Chordata, Euteleostomi, Mammalia and Primates. OTCSGs means the genes that can be detected only in rare samples with transcript evidence. PE3/4 genes means the genes encoding PE3 and PE4 proteins.

Figure S5. Evolutionary characteristics of genes encoding PE3 and PE4 proteins. OTCSGs means the genes that can be detected only in rare samples with transcript evidence. PE3/4 genes means the genes encoding PE3 and PE4 proteins. (A), Ages of genes encoding PE3 and PE4 proteins. All the protein-coding genes of $H$. sapiens are divided into 6 groups according to their evolutionary origin time, including the genes originating from the common ancestor of Opisthokonta, Bilateria, Chordata, Euteleostomi, Mammalia, and Primates. (B), Chromosomal evolutionary characteristics of genes encoding PE3 and PE4 proteins. Percentage of genes with different distribution among msHSBs are showed in different colors. (C), The percentage of paralogs in genes encoding PE3 and PE4 proteins. (D), Evolutionary rate of genes encoding PE3 and PE4 proteins. In each box, the central mark is the median, the edges of the box are the 25th and 75th percentiles ( $\mathrm{q} 1$ and $\mathrm{q} 3$ ), the whiskers extend to the most extreme data points not considered outliers, and outliers are plotted individually (red points).

Figure S6. The cumulative probability curve of the difference values of average order of PE3/4 and OTCSGs. The thin dotted line represents the boundary of difference values are equal to zero. PE3/4 genes are the genes encoding missing proteins without transcript evidence (See the 
Method section in the main text for detailed information). OTCSGs are the genes that can be detected at transcript level only in rare samples. If the difference value>0, it means the PE3/4 genes duplicated later than the OTCSGs in the same PGG (paralogous gene group). Otherwise, if the difference value $<0$, it means the PE3/4 genes duplicated earlier than the OTCSGs in the same PGG.

\section{Figure S7. Evolutionary characteristics of the proteins with extremely special} physical-chemical properties. (A), Chromosomal evolutionary characteristics of proteins with special physical and chemical properties. Percentage of genes with different distribution among msHSBs are showed in different colors. (B), The percentage of paralogs in proteins with special physical and chemical properties. (C), Evolutionary rate of proteins with special physical and chemical properties. In each box, the central mark is the median, the edges of the box are the 25th and 75th percentiles ( $\mathrm{q} 1$ and $\mathrm{q} 3$ ), the whiskers extend to the most extreme data points not considered outliers, and outliers are plotted individually (red points). 


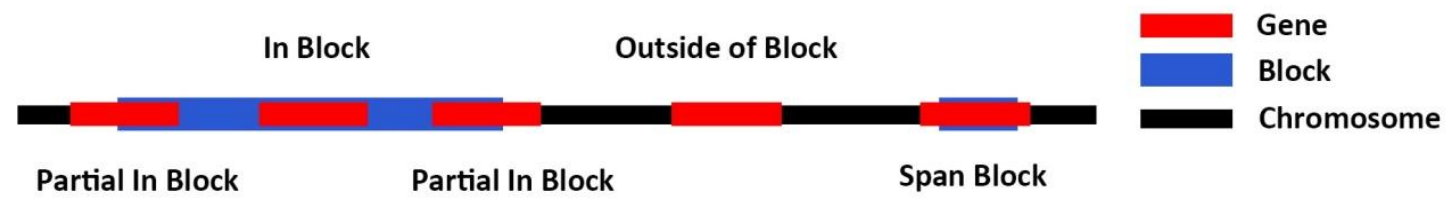

Figure S1. Schematic diagram for the relative locations of gene and block. 
A Telomeres in short arms

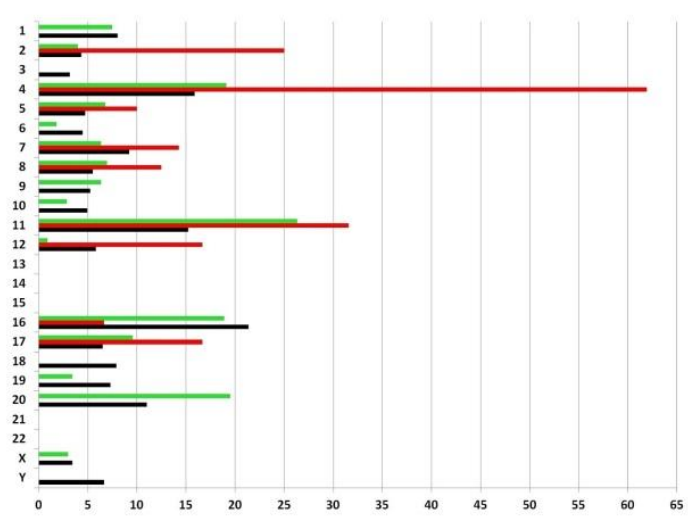

B Center of centromere

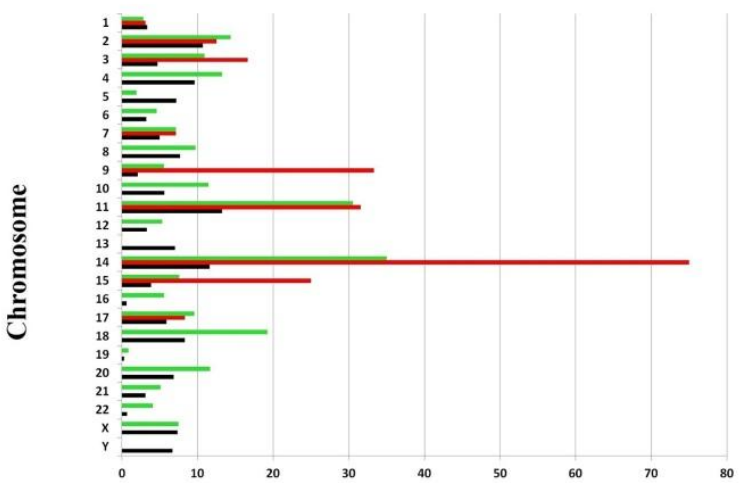

- PE3/4

- All

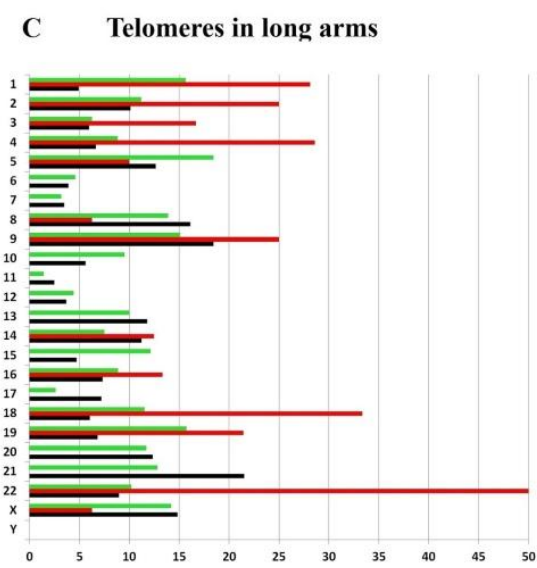

Percentage ( \% )

Figure S2. The percentages of the (missing-protein-encoding) genes closed to the telomeres in short arms (A), center of centromere (B) and telomeres in long arms (C) among all (missing-protein-encoding) genes in each chromosome. 


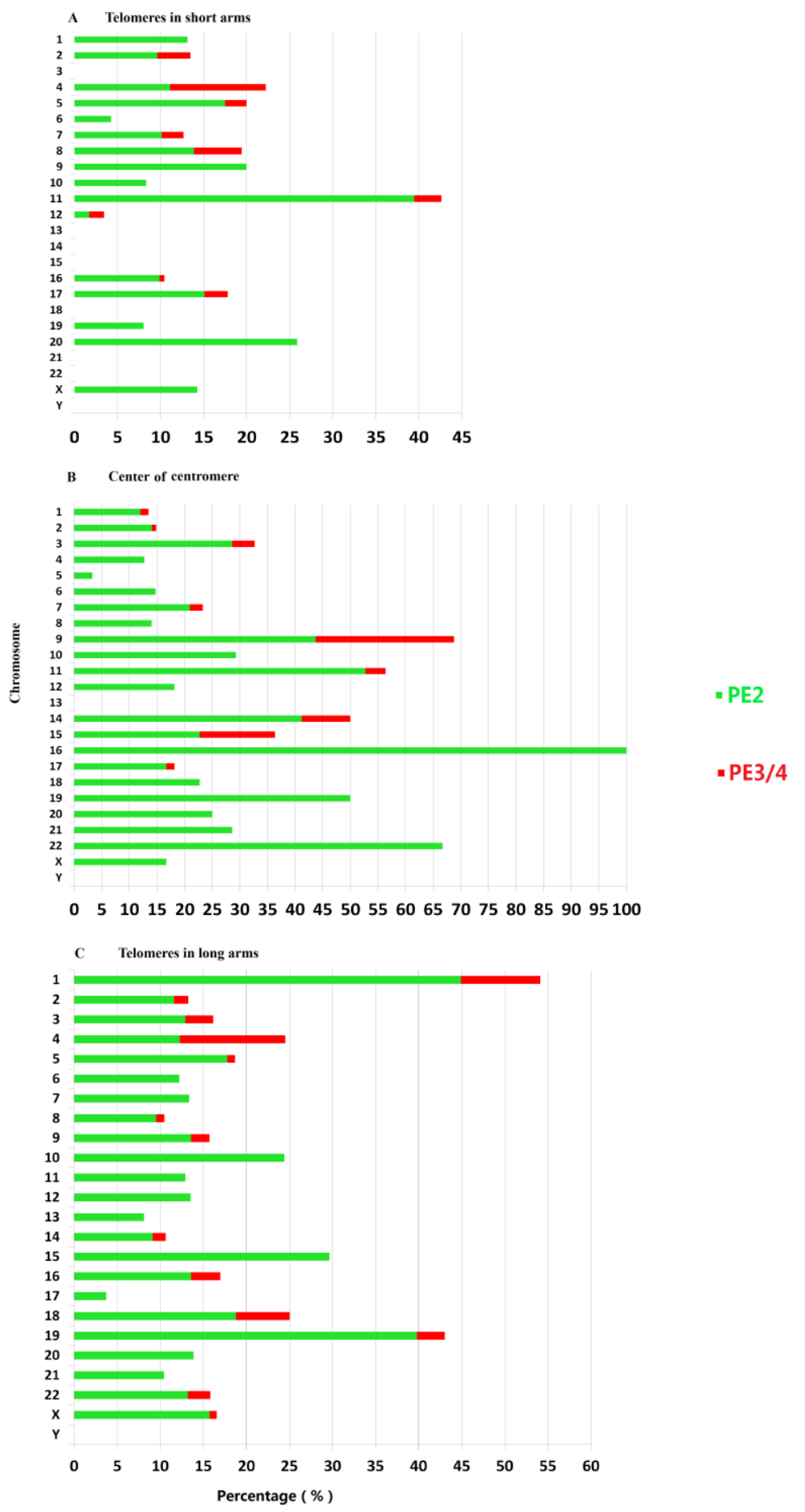

Figure S3. The percentages of the two types of missing-protein-encoding genes closed to the telomeres in short arms (A), center of centromere (B) and telomeres in long arms $(\mathrm{C})$ among all genes in each region. 


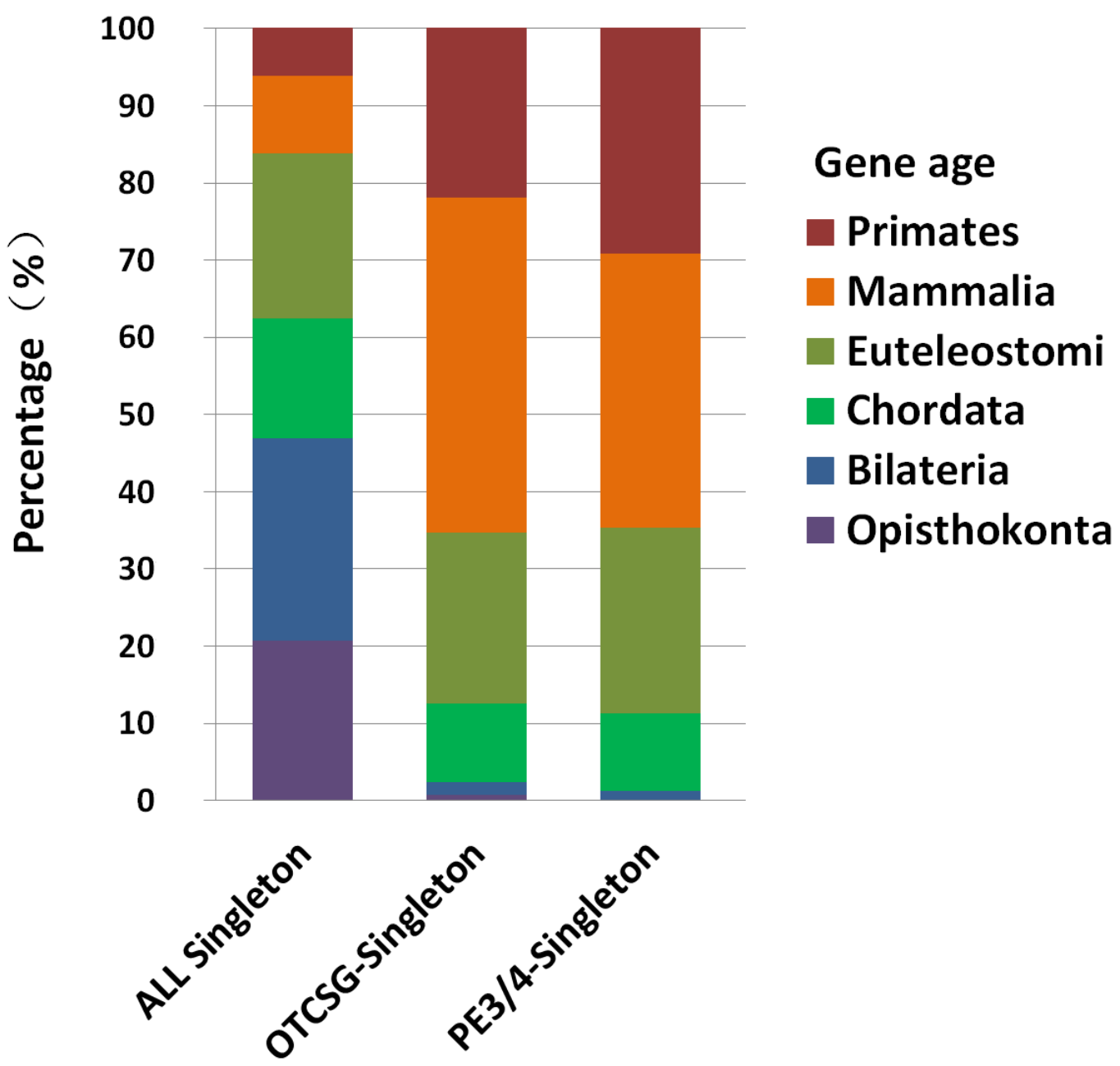

Figure S4. Ages of singletons. 

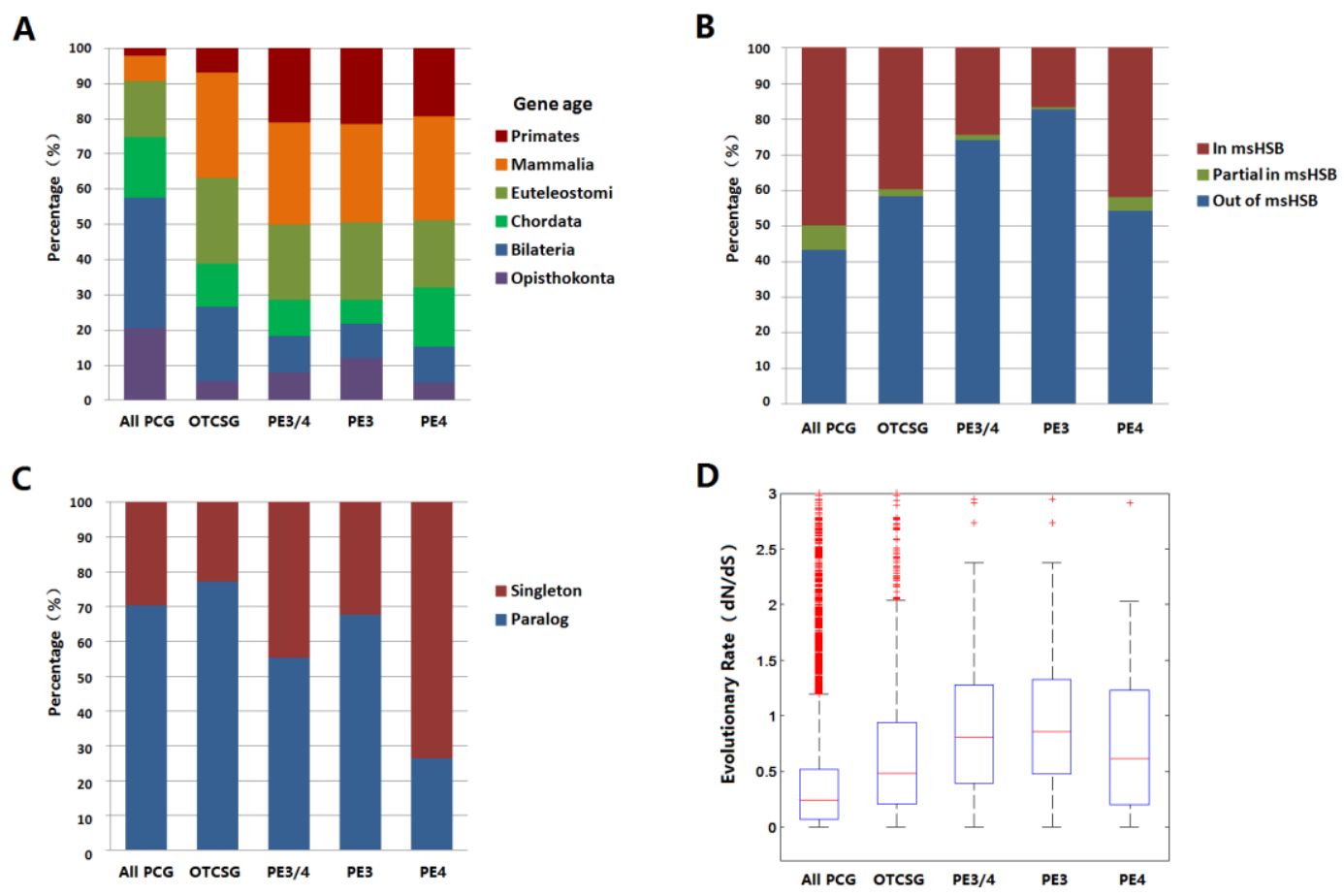

Figure S5. Evolutionary characteristics of genes encoding PE3 and PE4 proteins. 


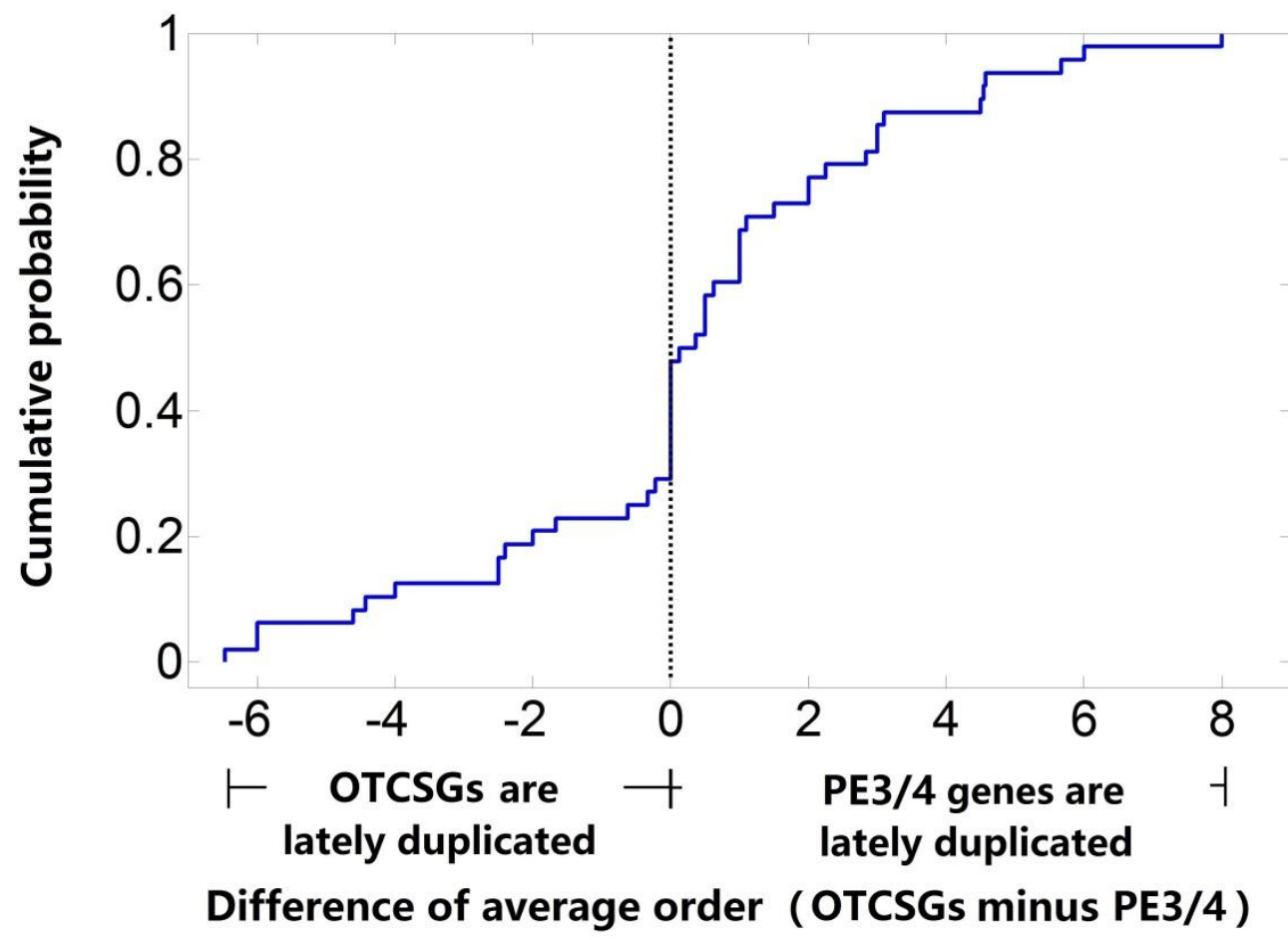

Figure S6. The cumulative probability curve of the difference values of average order of PE3/4 genes and OTCSGs. 

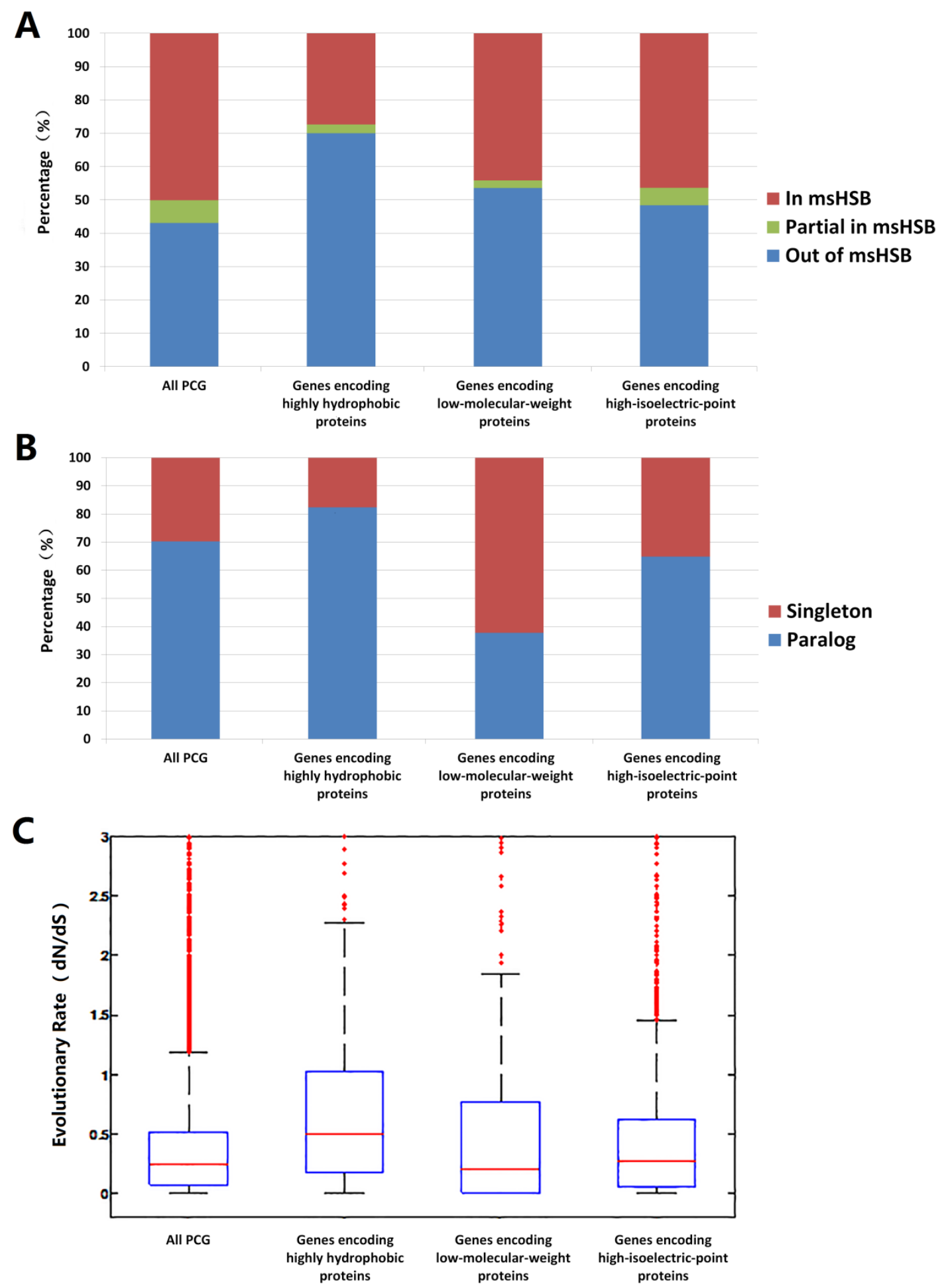

Figure S7. Evolutionary characteristics of the proteins with extremely special physical-chemical properties. 